\title{
Paying doctors for performance
}

\author{
Gérard de Pouvourville
}

Published online: 15 June 2012

(C) Springer-Verlag 2012

In health economics textbooks, most discussions around incentives for doctors have focused on a comparison of the three most current payment schemes: fee-for-service, capitation, and salary. In general, the scope of analysis is restricted to primary care physicians versus specialist care and care delivered by doctors in hospitals. The story is well known: fee-for-service will incentivize doctors to provide excess care and increase expenditure, but compared to the other schemes it has a positive effect on productivity and quality, although it is not always very clear how quality is defined. Moreover, it is important to specify whether the level of fee is imposed by the payer, or if doctors have some latitude in setting their prices. Capitation will lead to a better control over overall expenditure, but eventually to the detriment of quality, as measured by the unit time per visit and the number of visits. The claim of better control over expenditures can be challenged if - to minimize their effort-GPs systematically refer cases to specialists. Finally, the discussion around salary must take into account the organizational incentives that will inevitably complement the remuneration provided by the employer of the physician.

Such discussions have taken professional autonomy for granted, and as such the relevance of medical decisionsthe accuracy of the diagnosis and the relevance of the treatment decision-is left aside. Quality of care is measured mainly through the time spent with the patient and the reactivity to patient demands (in terms of time to access and personalization of the doctor/patient relationship). The underlying principle of service activity prevails: doctors,

G. de Pouvourville $(\square)$

ESSEC Business School, Avenue Bernard Hirsch, BP 50105 Cergy, 95021 Cergy Pontoise Cedex, France e-mail: pouvourville@essec.fr like other highly skilled professionals such as lawyers, have an ethical obligation to provide their best service, but because there is uncertainty over the outcome, they should not be obligated to provide a positive outcome. This led to ferocious criticism from George Bernard Shaw [1], who stated that you cannot trust somebody who makes money by making you feel unwell or cutting you into pieces. A reminder of the old Chinese tradition of paying doctors only if you are in good health is often used to disencourage the use of actual forms of payment. To complete the picture, another underlying assumption has been that payers, whether they are patients or third parties, are not in a capacity to observe medical practice due to ignorance (asymmetry of information due to expert knowledge) and the need for personalized care, thus leading to the rejection of any form of standardization of medical decision making.

This situation has changed dramatically in the last few decades under cost-containment pressure and with the development of information systems that allow medical practices to be monitored. Evidence-based medicine supports the argument that efficacy can be improved by avoiding unnecessary care. Thus, incentive schemes of all sorts targeting medical decisions have emerged in many countries. Amongst these, financial schemes have focused on primary care with the emergence of Health Maintenance Organizations in the USA in the 1980s. Indeed, the financial success of HMOs initially relied on reducing access to specialist care and hospitals. In initial staff models, such as that of Kaiser Permanente, this meant setting practice guidelines for employed physicians and other staff to follow. As early as in 1987, Alan Hillman [2, 3] started surveying and assessing the contractual arrangements signed between HMOs and individual practitioners, some of which included either bonuses or financial penalties based on the physician's experience and the financial results of 
the organizations. At that time, the aim was to reduce resource utilization, in particular rates of hospitalizations and of visits to physicians.

What has been growing is the salience of payment schemes directly targeting physician practice patterns, in order to incentivize physicians to adopt recommended care processes and in some cases to reward outcomes. Again, such recommended processes were already implemented in managed care organizations in the USA under the broad label of disease management programs, which actually targeted both physicians and patients. There are also many initiatives that aim to promote integrated primary care services, like those in Germany [4]. The change with new performance-based payment schemes is that the former could be considered to be standard operating procedures set at an organizational level, whereas new payment schemes (as we shall see) are direct financial incentives for independent practitioners that target clinical pathways and in some cases outcomes.

It is no surprise that the most varied and extensive schemes have emerged in the USA, where all insurers struggle to contain costs while maintaining a high level of quality of care. According to a review prepared for the French government by Bras and Duhamel in 2008 [5], the number of P4P programs rose from 39 in 2003 to 147 by the end of 2007, with the number expected to rise to 160 by the end of 2009. All of the programs reviewed mainly targeted general practitioners. Indicators cover clinical efficacy and safety, implementation of preventative actions, drug usage, patient satisfaction, and the use of new information technologies (NIT).

In Europe, much publicity has been given to the program implemented in 2004 by the NHS in the context of the overall Quality and Outcomes Framework (QOF) [6]. The NHS had already used financial incentives in the past, for example to improve the coverage for screening programs, but QOF should be viewed as a comprehensive effort to incentivize physicians to improve the quality of their practice in multiple dimensions. The general traits of QOF are well known: in the original scheme, a set of indicators were defined through negotiation with the British Medical Association that covered clinical and organizational indicators, patient satisfaction, and specific screening and patient management programs. The incentive is an annual bonus paid to the physician according to the level of performance observed, based on the cumulative score on all dimensions.

France has recently followed the route taken by the NHS and implemented an individual pay-for-performance contract on a voluntary basis. According to this contract, called CAPI (Contract to Improve Individual Practices), GPs get a bonus if they met performance targets based on the quality of diabetic patient follow-up, on screening coverage, and on specific drug prescription targets. By the end of 2009, some $20 \%$ of GPS had signed the contract. Since then, the CAPI has been included in the new general agreement signed between the Sickness Fund and the physicians' unions, which means that the latter will be included in the future when defining indicators, new targets, and bonuses. The CAPI can be considered a mini-QOF with less extensive coverage.

What are the main issues raised by such schemes from an economist's perspective? First, we must now revise the usual premise of health economics-asymmetry of information between the payer and the provider. Under this premise, professional autonomy was granted without discussion to physicians due to their long training and expert knowledge, due to the variability associated with individual cases, and due to the uncertainty in treatment outcomes. This provided the basis for professional autonomy, selfregulation by the profession, and traditional economic models of physician behavior; either maximizing income under the constraints of professional ethics and deontological rules or maximizing a utility function with three arguments: income, professional pride, and dedication to the patients' needs. Now payers can observe medical practices, assess them against norms set by the professional themselves (EBM), and require that they use resources efficiently, from their perspective. Professionals usually claim that they are held by an obligation of means, not outcomes, and that they are free to choose the resources that they will use to deliver their services. Payment per performance implies that they should be paid according to a predefined process of care that they must comply with. In general, this is based on the assumption that if the process is complied with, then favorable outcomes for patients will have a higher probability of being reached.

Second, paying a bonus per performance reflects the assumption that quality of care is not at expected standards because physicians consider, under current payment schemes, that the cost of the effort is lower than its financial yield compared to other actions they could take; for example, increasing their activity under fee-for-service or increasing their list of patients under capitation. There may be other reasons for an observed heterogeneity of performance, like adaptations to perceived preferences of patients who may not be willing to accept increased monitoring and counseling, acceptance of practice guidelines, or-in the case of a preference for branded prescriptions over generics in a given class-the belief that branded drugs are more potent and more beneficial to the patient. Recent research work has also stressed the idea that physicians' behavior can be influenced by both "intrinsic" and "extrinsic" motivations. "Intrinsic" motivations are another way of formalizing the altruistic sense of service to the patient and the professional pride that physicians take 
in benevolently providing their time and skills without considering payment. Intrinsic motivations are related to activities that physicians perform for the direct satisfaction they provide, with no need for reward or fear of a penalty. "Extrinsic" motivations are actually responses to external incentives, whether regulatory or financial. Deontology may be self-accepted by physicians, but it is also enforced through professional regulations, and can lead to legal actions, so they may well belong to the registry of extrinsic motivations, whereas dedicating extra time to complex cases on a humanistic basis beyond what would be considered the usual, state-of-the-art service belongs to the sphere of intrinsic services.

Under this framework, physicians could consider that adding financial incentives, even if motivated by an improvement in the quality of care, is a denial of their professional and personal humanistic values. A financial incentive to improve quality could also be perceived as an implicit value judgment on their actual quality of care. Thus, financial incentives could either be rejected, leading to a refusal to play the game of performance-based payment with an inherent risk of not receiving the bonus, or there could be a "crowding out" effect. Dedicating time and effort to earn the bonus could decrease the time spent "without counting," thus depriving patients of the potential benefits of a highly dedicated physician. However, one could also argue that some physicians who already perform well according to the goals set by the payer may consider the bonus to be a legitimate reward for the quality of their work that is not acknowledged in a uniform payment scheme. This theoretical framework challenges the traditional egoistic view of altruism that economists usually adopt, in that it stresses a difference in nature between the two sets of motivations: satisfaction derived from exogenous rewards is not of the same nature as satisfaction derived from self-pride. Thus, intrinsic motivations could be independent from extrinsic motivations and lead to crowding out. Proponents of this theory then argue that P4P may well transform physicians into "pure" economic agents, forgetting their sense of dedication to the patients' needs, both clinical and humanistic. Because the patients' adherence to disease management programs or to health promotion is a key success factor, and a partly exogenous one, there also may be an incentive for physicians to select "good" patients (i.e., those with a higher level of education and better compliance). In this argument, it may be necessary to differentiate incentives targeting quality of care, which refer to a model of clinical excellence, from incentives targeting efficiency (for example the percentage of generic prescriptions in a given class), about which physicians may raise ethical concerns. However, this distinction may be fallacious, since quality of care guidelines are not economically neutral.
An empirical test of this theory is difficult to realize, since it requires defining observable practices or activities as being mainly intrinsic. However, this framework points to another criticism of P4P. By linking financial rewards to performance in a limited number of domains, and depending on the level of the financial incentives at stake, this could lead to less time and effort being dedicated to activities and domains that are not covered by the scheme. Any assessment of this eviction effect requires an evaluation of the supplementary effort that must be invested in terms of time and intensity to achieve a satisfying level of performance. The USA experience suggest that including incentives for the use of new information technologies has a synergistic effect, in that it allows the productivity of doctors in relation to the management of patients to be improved. In particular, this may also reduce the time needed to report results to the payer-an issue that has been raised by French GPs who have adopted the new contract.

This being said, it is not clear whether improving one's performance will result in more work overall. The development of P4P was motivated by the observable heterogeneity in medical practice, as measured by payers through claims data or specific surveys. However, little is known about the relationship between observed quality of performance, overall workload, and level of income. It is thus possible that some physicians perform well according to quality standards at no extra expense in terms of their working time and revenues, in contrast to other colleagues. Thus, the challenge may well be for physicians to reengineer their practices in order to reach a new permanent state. Incentives should then be considered a subsidy to pay for the time on the learning curve.

The last issue to be discussed is the required level of incentives. According to the 2008 UK Review Body on Doctors' and Dentists' Remuneration [7], QOF increased GP incomes by $25 \%$ on average (by $£ 20,000$ ). Considering the variety of schemes in the USA, it is more difficult to estimate the percentage of doctors' incomes linked to P4P schemes. In France, the National Sickness Fund has recently announced an annual average bonus of $€ 4,500$ per year, whereas the average income was $€ 71,300$. In an interview for the journal Le Quotidien du Médecin, [8] the Director General of UNCAM (the National Union of Sickness Funds) predicted that the payment per performance bonus could reach 15-20\% of GPs' income in the next few years.

When choosing the level of incentives, it is important to take into account the following factors. First, payments should be at least equal (or perceived to be equal by physicians) to the present hourly rate of their usual activities (minimum reservation price); second, the total potential income bonus should also be high enough for GPs to 
consider joining, even if the unit hourly rate is perceived to be satisfactory; finally, this hourly unit rate and the total amount should not be too high, in order to avoid an exclusion effect on other activities not covered by the scheme.

A complementary issue is that of the potential for freeriding: some physicians will already be close to achieving the maximum goals, so they need to invest little or no extra effort to gain the bonus. This issue could be circumvented by the payer if he/she has previous knowledge of the existing level of performance of the physician, but it may be politically difficult in the first years of such a program to adjust rewards to existing levels. The annual revision of such schemes has also been discussed: the UK experience suggests that most physicians rapidly reach high scores for given targets, meaning that targets must evolve over time and thus questioning the long-term sustainability of the scheme.

Finally, one could argue that the amount dedicated to improving performance should also be related to its expected cost-effectiveness. Pragmatically, it may be difficult to perform such an ex ante calculation, considering the variety of targets in existing schemes. In particular, it is difficult to assess the cost-effectiveness of improving the structural quality of GP practices. In France, it was possible to estimate the cost savings related to the prescription of generics, and the Sickness Fund anticipates a reduction in hospital admissions for chronic diseases such as diabetes and hypertension, but this will only be observable once the scheme has been implemented for a few years and at a significant level.

A full review of the assessment of ongoing payment schemes is beyond the scope of this editorial. Whatever conclusions are drawn, a reasonable guess is that they will be changed incrementally and refined, which has already been the case for QOF and CAPI. Indeed, the more substantial they are in terms of additional revenue for physicians, the more difficult it will be to pull out without an income neutral alternative. Whatever alternative is designed, there is no going back to a situation of blind payers: the information systems and management technologies used by physicians will improve, giving payers more latitude to ask for quality and efficiency counterparts to service payment. It is also predictable that schemes will give progressively more weight to outcome indicators relative to structure and process. Since primary care practice will be increasingly integrated into pluriprofessional groups, payers' incentives will target organizations, and leave it up to them to implement incentives for their staff. If this is the case, then studies of the impact of payment schemes on physicians will move from the individual to the organizational level. Finally, it may be that in the future, patients will also require information on the comparative quality performances of various health care services, thus introducing another source of pressure on health care professionals. Will this be detrimental to intrinsic motivations, or will these change over time?

\section{References}

1. Shaw GB.: The doctor's dilemma. London: Constable (1908)

2. Hillman, A.: Financial incentives for physicians in HMOs. N. Engl. J. Med. 317, 1743-1748 (1987)

3. Hillman, A., Pauly, M., Kerstein, J.J.: How do financial incentives affect physicians' clinical decisions and the financial performance of health maintenance organizations? N. Engl. J. Med. 321, 86-92 (1989)

4. Greb, S., Focke, A., Hessel, F., Wasem, J.: Financial incentives for disease management programmes and integrated care in German social health insurance. Health Policy 78(2-3), 295-305 (2006)

5. Bras, P.L., Duhamel, G.: Rémunérer les médecins selon leurs performances : les enseignements des expériences étrangères (Paying doctors according to their performances: lessons from abroad) (report). Paris: General Inspection of Social Affairs (2008)

6. NHS: The Quality and Outcomes Framework 2004/2005. http://www.ic.nhs.uk/statistics-and-data-collections/audits-and-per formance/the-quality-and-outcomes-framework/the-quality-and-out comes-framework-2004-05. Accessed April 2012

7. Review Body on Doctors and Dentists Remuneration: Thirty seventh report 2008, Cm 7327. London: The Stationery Office (2008). http://www.dh.gov.uk/en/Publicationsandstatistics/Publi cations/PublicationsPolicyAndGuidance/DH_080117

8. van Roeckeghem, F.: From CAPI to P4P: "It is in the doctors' best interest to join" (interview). Le Quotidien du Médecin, 09/21/2011 